\title{
Use of prednisolone to prevent the development of immunity reconstitution syndrome in patients with multidrug-resistant tuberculosis
}

\author{
Matvyeyeva S.L.
}

Kharkiv National Medical University, Kharkiv, Ukraine

Inflammatory reconstitution of immune syndrome (IRIS) is an immunological reaction characterized by recurrent or new inflammatory signs of tuberculosis (TB) that occur shortly after antiretroviral therapy (ART) in $18 \%$ of patients infected with human immunodeficiency virus (HIV). Features of the syndrome include recurrent symptoms: fever, lymphadenitis and the spread of pulmonary infiltrates on radiography. Low CD4 levels and a short interval between the onset of ART increase the risk of developing TB-associated immune system recovery syndrome. Clinical trials show that early onset of ART results in higher survival rates than ART initiated approximately 8 weeks after the start of TB treatment in patients with a CD4 count of 50 cells $/ \mu$. These findings are in line with the recommendations of the World Health Organization, which emphasize the acceleration of the onset of ART in patients with TB and low CD4 levels. However, despite the advantage of survival, early administration of ART more than increases the risk of developing IRIS associated with TB. There is no evidencebased strategy for the prevention of IRIS associated with TB. Studies evaluating adjuvant glucocorticoids for the treatment of various forms of TB have shown reduced mortality among TB patients and fewer complications among TB patients. The aim of the study was to evaluate the prophylactic use of prednisolone to safely reduce the incidence of TB-associated IRIS in patients with multidrug resistant TB (MDR TB) is at high risk of developing the syndrome. We observed HIV-infected patients who started ART (and had not previously received ART) and started TB treatment within 30 days before the start of ART and had a CD4 count of 50 cells/ $\mu$ l. Patients received either standard therapy with prednisolone (at a dose of $40 \mathrm{mg}$ per day for 14 days, then $20 \mathrm{mg}$ per day for 14 days) or only standard therapy without prednisolone. The main endpoint was the development of TB associated with TB within 12 weeks after the onset of ART. Among the 64 patients observed, the mean age was 36 years, $60 \%$ were male, and $73 \%$ had microbiologically confirmed TB; the median amount of CD4 was 49 cells/ $\mu$ l, and the median viral load was 5.5 log 10 copies/ $\mu$ l. Patients were divided into 2 groups. Each group included 32 patients with MDR TB / HIV co-infection. IRIS associated with TB was diagnosed in 10 patients (31.25\%) in the prednisolone group and in 17 (53.14 \%) in the control group $(p<001)$. Thus, prednisolone treatment of coinfected patients with MDR TB for the first 4 weeks after the onset of HIV-related ART resulted in a lower incidence of TB-associated IRIS than in the control group, with no evidence of an increased risk of severe infections or cancer. 